Draft VERSion April 10, 2017

Preprint typeset using LATEX style AASTeX6 v. 1.0

\title{
WORLDS WITHOUT MOONS: EXOMOON CONSTRAINTS FOR COMPACT PLANETARY SYSTEMS
}

\author{
STEPhen R. KANE
}

Department of Physics \& Astronomy, San Francisco State University, 1600 Holloway Avenue, San Francisco, CA 94132, USA

\begin{abstract}
One of the primary surprises of exoplanet detections has been the discovery of compact planetary systems, whereby numerous planets reside within $\sim 0.5 \mathrm{AU}$ of the host star. Many of these kinds of systems have been discovered in recent years, indicating that they are a fairly common orbital architecture. Of particular interest are those systems for which the host star is low-mass, thus potentially enabling one or more of the planets to lie within the Habitable Zone of the host star. One of the contributors to the habitability of the Earth is the presence of a substantial moon whose tidal effects can stabilize axial tilt variations and increase the rate of tidal pool formation. Here we explore the constraints on the presence of moons for planets in compact systems based on Hill radii and Roche limit considerations. We apply these constraints to the TRAPPIST-1 system and demonstrate that most of the planets are very likely to be worlds without moons.
\end{abstract}

Keywords: astrobiology - planetary systems - stars: individual (TRAPPIST-1)

\section{INTRODUCTION}

Over the past few decades, the field of exoplanets has provided no shortage of orbital architectures for which we have no analog in our own planetary system. These include hot Jupiters, massive planets in eccentric orbits, circumbinary planets, and compact planetary systems. The initial windfall of compact planetary systems came from the early results of the Kepler mission. The bias of the transit method towards short orbital periods (Kane \& von Braun 2008) meant that these compact systems exist in the region of parameter-space where Kepler had the strongest sensitivity. However, the frequently faint host stars also meant that masses could not easily be measured from precision radial velocities. Fortunately the short period of the compact system planets and the precision of the Kepler photometry allowed the measurement of the masses through Transit Timing Variations (TTVs) (Miralda-Escudé 2002; Agol et al. 2005; Holman \& Murray 2005). The measurements of the planetary masses allow, amongst other things, exploration of the orbital stability of these systems (Raymond et al. 2009; Funk et al. 2010; Zhang et al. 2013; Hands et al. 2014; Becker \& Adams 2017).

Notable examples of compact planetary systems with measured masses are Kepler-11 (Lissauer et al. 2011, 2013) and Kepler-80 (Xie 2013; MacDonald et al. 2016). The recently discovered TRAPPIST-1 system provides

skane@sfsu.edu a compact orbital architecture where some of the planets appear to occupy the Habitable Zone (HZ) of the host star. Initial observations uncovered three terrestrial planets transiting the faint ultracool dwarf star (Gillon et al. 2016). Continued observations using Spitzer revealed an additional four terrestrial transiting planets, including TTVs that allowed the masses of the planets to be determined (Gillon et al. 2017). A longer time baseline of photometry provided by the $\mathrm{K}$ 2 extension of the Kepler mission confirmed the orbital period of the outer planet that placed the orbit near the TRAPPIST-1 snow line (Luger et al. 2017).

Much has been written about the detection of exomoons and their potential habitability (Reynolds et al. 1987; Williams et al. 1997; Weidner \& Horne 2010; Forgan \& Kipping 2013; Hinkel \& Kane 2013; Heller \& Barnes 2013; Heller et al. 2014; Kipping et al. 2015). It is also commonly held that the presence of a moon with substantial mass played a key role in Earth's habitability through obliquity stabilization. Early work by Lasker et al. (1993) indicated that a moonless Earth would have extreme variations in obliquity resulting in dramatic climate changes. A study by Tomasella et al. (1996) expanded the chaotic obliquity calculations by included the effects of the tidal expansion of the moon. Further simulations by Lissauer et al. (2012) and Li et al. (2014) demonstrated that the Moon does indeed stabilize the Earth's obliquity, though not at the previously determined amplitude and thus a moonless Earth does not necessarily preclude habitability.

Here we explore the role of orbital architecture and 
planetary masses and radii on the ability of the planets to harbor exomoons. We show that gravitational influence of planets in compact planetary systems places severe constraints on the presence of exomoons and we use the TRAPPIST-1 system as an example. In Section 2, we quantify the limits imposed on exomoon orbits by the Hill radius and Roche limits of a planet. In Section 3, we construct analytical limits for exomoon stability regions in the context of compact planetary system architectures. Section 4 applies these constraints to the TRAPPIST-1 system and demonstrates why many of the TRAPPIST-1 planets cannot harbor moons. We provide discussion and concluding remarks in Section 5.

\section{HILL RADIUS AND ROCHE LIMIT}

The two main considerations for the region in which a moon can be maintained in orbit around a planet are the Hill radius, which defines the outer limit of the region, and the Roche limit, which defines the inner limit (Kipping 2009; Heller 2012). The Hill radius, $R_{H}$, is given by

$$
R_{H}=a_{p}\left(\frac{M_{p}}{3 M_{\star}}\right)^{\frac{1}{3}}
$$

where $a_{p}$ is the planetary semi-major axis, $M_{p}$ is the planetary mass, and $M_{\star}$ is the stellar mass. The Roche limit, $R_{R}$, is given by

$$
R_{R} \simeq 2.44 R_{p}\left(\frac{\rho_{p}}{\rho_{m}}\right)^{\frac{1}{3}}
$$

where $R_{p}$ is the planetary radius, $\rho_{p}$ is the planetary density, and $\rho_{m}$ is the moon density. For example, the Hill radius of the Earth is $0.01 \mathrm{AU}$ and the Roche limit is $1.26 \times 10^{-4} \mathrm{AU}$, a factor of $\sim 78.5$ difference. For an Earth-analog in a compact system with a semi-major axis of $0.05 \mathrm{AU}$, the Hill radius shrinks to $6 \times 10^{-4} \mathrm{AU}$ which results in a factor of $\sim 4.5$ difference with the Roche limit.

Given that the radius of the Hill sphere is a theoretical approximation, the practical outer limit of a stable orbit for a moon can be significantly smaller due other perturbation effects, such as those originating from the host star. As such, the outer limit may be described as $\chi R_{H}$ where $\chi$ is a reduction factor due to the above described effects (Holman \& Wiegert 1999). Further perturbation sources are other bodies in the system, particularly planetary bodies (Gong et al. 2013; Payne et al. 2013). Furthermore, formation processes play a role in the destabilization of moons, such as their potential for resonant removal during planetary migration (Spalding et al. 2016). For example, Barnes \& O'Brien (2002) and Kipping (2009) estimate $\chi \approx 1 / 3$, effectively reducing the outer limit of a stable moon orbit to $1 / 3 \times R_{H}$. An additional process that will reduce the

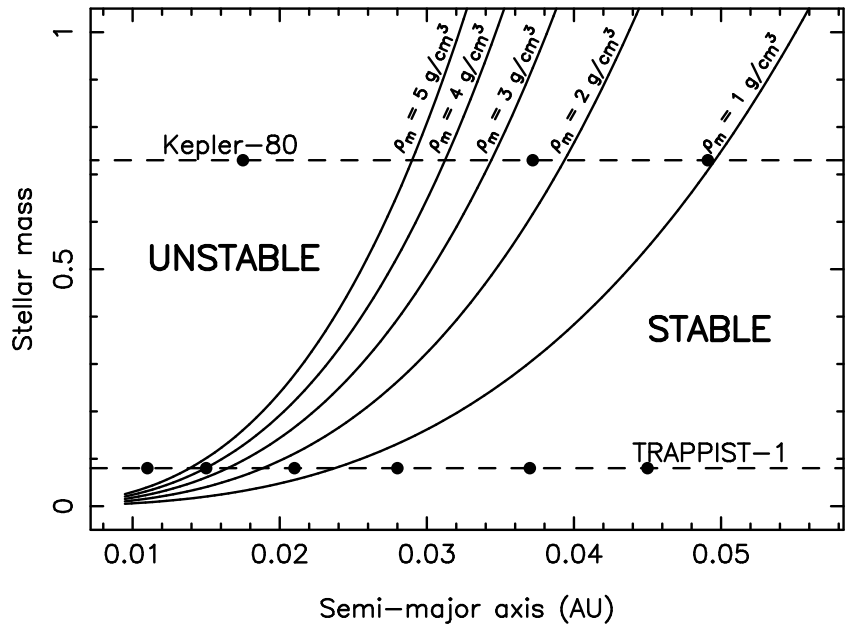

Figure 1. The locations of equality between the Hill radius and Roche limit (see Equation 5) for five different mean densities of a potential moon. Stable moon orbits cannot exist above the curve for a given density. For the purposes of these calculations, we adopt a reduction factor of $\chi=1 / 3$. The horizontal dashed lines indicate the stellar masses for two example compact planetary systems, Kepler80 and TRAPPIST-1, with the locations of the planets shown on each line.

size of the allowed region for long-term moon orbital stability is the evolution of the orbit through tidal interactions with the planet (Sasaki \& Barnes 2014), effectively resulting in an exchange of angular momentum. The inward or outward direction of the moon migration depends upon the ratio of the orbital period to the rotation period of the planet (Barnes \& O'Brien 2002) and also if the orbit is prograde or retrograde (Domingos et al. 2006). Note that the semi-major axis of the Moon's orbit is $\sim 26 \%$ of the Earth's Hill radius, which is close to the $\chi \approx 1 / 3$ criteria mentioned above. For comparison, the outermost of the Galilean moons, Callisto, has a semi-major axis that is $\sim 3 \%$ of Jupiter's Hill radius. Clearly the effective size of the Hill sphere for planets in compact systems can be greatly reduced by the properties of the local environment.

\section{EXOMOON CONSTRAINTS FOR COMPACT PLANETARY SYSTEMS}

Here we consider the Hill and Roche limit radii described in Section 2 in the extreme environments of compact planetary systems. Within the orbital regime of these systems, the gravitational perturbations are dominated by the host star rather than the planets, enabling stability through the shrinking of the planetary Hill spheres (Namouni 2010). An upper limit of the presence of moons may be determined by evaluated conditions where $\chi R_{H}=R_{R}$. Using Equations 1 and 2, we find the following relationship:

$$
\chi a_{p}\left(\frac{M_{p}}{3 M_{\star}}\right)^{\frac{1}{3}}=2.44 R_{p}\left(\frac{1}{\rho_{m}} \frac{M_{p}}{\frac{4}{3} \pi R_{p}^{3}}\right)^{\frac{1}{3}}
$$


which is simplified as

$$
\rho_{m}=10.4 \frac{M_{\star}}{\left(\chi a_{p}\right)^{3}}
$$

A more convenient form of the relationship is as follows:

$$
\left(\frac{\rho_{m}}{\mathrm{~g} / \mathrm{cm}^{3}}\right)=6.18 \times 10^{-6} \chi^{-3}\left(\frac{M_{\star}}{M_{\odot}}\right)\left(\frac{a_{p}}{1 \mathrm{AU}}\right)^{-3}
$$

Equation 5 is plotted in Figure 1 for densities ranging from $1 \mathrm{~g} / \mathrm{cm}^{3}$ to $5 \mathrm{~g} / \mathrm{cm}^{3}$, where we have assumed a Hill radius reduction factor of $\chi=1 / 3$. Horizontal dashed lines indicate the stellar masses for the Kepler$80\left(0.73 M_{\odot}\right)$ and TRAPPIST-1 $\left(0.0802 M_{\odot}\right)$ host stars, the planets of which largely fall within the range of semimajor axes shown (MacDonald et al. 2016; Gillon et al. 2017). Since the solid curves represent the boundary where $\chi R_{H}=R_{R}$, moons of the given density cannot exist within regions of parameter space that lie above the curve. For example, no planet in the Kepler- 80 system within $0.035 \mathrm{AU}$ of the host star can ever host a moon with a density $\leq 3 \mathrm{~g} / \mathrm{cm}^{3}$. The cubic nature of the relationship described in Equation 5 does ensure that the concise exclusion of moons predominantly affects those planets in the very small semi-major axis regime. An interesting possibility is that the moon constraints for compact systems may still allow the presence of ring systems (Barnes \& Fortney 2004; Zuluaga et al. 2015), particularly considering the relatively long persistence of debris disks around low-mass stars (Plavchan et al. 2009). Such rings around terrestrial planets are likely to have low optical depth and not readily detectable from transit photometry when they are coplanar with the inclination of the planetary orbits.

Table 1. TRAPPIST-1 planetary Hill radii and Roche limits

\begin{tabular}{ccccccc}
\hline \hline Planet & $M_{p}\left(M_{\oplus}\right)$ & $R_{p}\left(R_{\oplus}\right)$ & $a(\mathrm{AU})$ & $R_{H}\left(10^{-3} \mathrm{AU}\right)$ & $R_{R}\left(10^{-3} \mathrm{AU}\right)$ & $R_{H} / R_{R}$ \\
\hline TRAPPIST-1 b & 0.85 & 1.086 & 0.011 & 0.244 & 0.120 & 2.04 \\
TRAPPIST-1 c & 1.38 & 1.056 & 0.015 & 0.393 & 0.141 & 2.79 \\
TRAPPIST-1 d & 0.41 & 0.772 & 0.021 & 0.370 & 0.094 & 3.94 \\
TRAPPIST-1 e & 0.62 & 0.918 & 0.028 & 0.557 & 0.108 & 5.17 \\
TRAPPIST-1 f & 0.68 & 1.045 & 0.037 & 0.756 & 0.111 & 6.80 \\
TRAPPIST-1 g & 1.34 & 1.127 & 0.045 & 1.154 & 0.139 & 8.28 \\
TRAPPIST-1 h & 0.31 & 0.715 & 0.060 & 0.936 & 0.086 & 10.86 \\
\hline
\end{tabular}

Note-Planetary masses, radii, and semi-major axes are from Gillon et al. (2017).

\section{NO MOONS IN THE TRAPPIST-1 SYSTEM?}

The TRAPPIST-1 system is an exceptional case of compact planetary systems due to the extremely low mass of the host star. The combination of transits and TTVs provide radii and masses for the planets, allowing us to calculate the Hill radii and Roche limits for each planet (see Section 2). Currently the outer planet (planet $\mathrm{h}$ ) does not have a reliable mass estimate, and so we used the mean density of the other six planets of $\sim 0.84 \rho_{\oplus}$ to determine a mass estimate of $0.31 M_{\oplus}$. The resulting calculations for all seven planets are shown in Table 1. Also shown are the ratios of the Hill radii and Roche limits, where a Hill radius reduction factor of unity has been used and a moon density of $3 \mathrm{~g} / \mathrm{cm}^{3}$ adopted for the Roche limit. For comparison, the mean density of the Galilean moons is $2.6 \mathrm{~g} / \mathrm{cm}^{3}$ and the mean density of the Earth's moon is $3.3 \mathrm{~g} / \mathrm{cm}^{3}$. The ratios in the final column of Table 1 show that, even for the case of $\chi=1$, the difference between the Hill radii and Roche limits for the inner planets is remarkably small, similar to the case for the Earth-analog at $0.05 \mathrm{AU}$ described in Section 2.

Shown in Figure 2 are moon exclusion boundaries (solid lines) for the TRAPPIST-1 system as a function of mean density and semi-major axis, with vertical dotted lines indicating the location of the six innermost planets. The shaded regions show the extent of the HZ for TRAPPIST-1, where we used the TRAPPIST1 luminosity $\left(0.000524 L_{\odot}\right)$ and effective temperature (2559 K) provided by Gillon et al. (2017), along with the HZ boundary equations of Kopparapu et al. (2013, 2014). The shaded regions correspond to the "conservative" (light-gray, 0.024-0.049 AU) and "optimistic" (dark-gray, 0.019-0.051 AU) HZ boundaries, the definitions of which depends upon assumptions regarding the longevity of liquid water on the surfaces of Venus and Mars. Although the full extent of the perturbations 


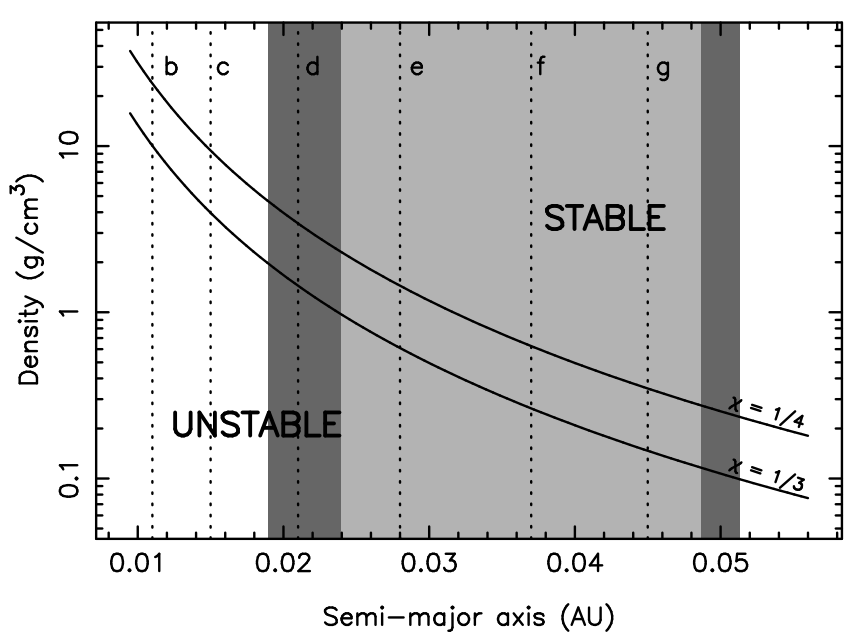

Figure 2. The allowed moon density as a function of semimajor axis for the TRAPPIST- 1 system and for Hill radii reduction factors of $\chi=1 / 3$ and $\chi=1 / 4$. Stable moon orbits cannot exist below each of the curves. The vertical dotted lines indicate the location of the six innermost TRAPPIST-1 planets. The light-gray shaded region shows the extent of the conservative HZ, and the dark-gray shaded region represents the optimistic extension to the HZ.

to potential moons is not quantified, the existence of resonant chains of planetary orbits (Gillon et al. 2017) is expected to increase the amplitude of such perturbations. Therefore, we have used Hill radii reduction factors of $\chi=1 / 3$ and $\chi=1 / 4$ in the figure. In this case, the exclusion boundaries imply that the parameter space below the curve show where stable orbits of moons are not possible. For example, the $\chi=1 / 4$ curve indicates that planets $\mathrm{b}-\mathrm{e}$ cannot host moons of reasonable $\left(\lesssim 3 \mathrm{~g} / \mathrm{cm}^{3}\right)$ mean densities. As noted at the end of Section 2, the Hill radii reduction factors do not include the effects of moon orbital migration due to a tidal exchange of angular momentum (Sasaki et al. 2012). Thus the region for which each planet can sustain a moon in a stable orbit over long time periods is further reduced such that it is highly unlikely that any of the planets represented in Figure 2 host moons. Properties of the host star, such as activity and rotation rate, suggest that the planetary system may be relatively young (Gillon et al. 2016). The outer planets may therefore currently hold moons in temporarily stable locations although, as noted earlier, the process of migration into regions of resonance trapping may have stripped them already (Spalding et al. 2016).

\section{CONCLUSIONS}

Although the discovery of compact planetary systems was initially a surprising outcome of exoplanet observations, it now seems as though they may be a quite common occurrence. In many cases, their architectures have been compared to that of the Jovian system leading to speculation of similar formation mechanisms
(Kane et al. 2013). An aspect of particular interest is the presence of compact systems around low-mass stars where the chances of temperate terrestrial planets are greatly enhanced. The TRAPPIST-1 system is an ideal example of such a system, with three planets within the conservative $\mathrm{HZ}$ and four within the optimistic HZ. This has resulted in the postulated habitability of the TRAPPIST-1 planets (Gillon et al. 2017), including detailed models of the planetary atmospheres (Wolf 2017). The presence of moons can have a positive impact on habitability, such as promoting tidal pools and stabilizing rotational obliquity. Insofar as habitability is reduced by a lack of moons, compact planetary systems such as TRAPPIST-1 may suffer due to the constraints on the presence of moons outlined in this work. As shown, a dominant deciding factor is the mean density of the moon which depends on whether they formed in situ around the planet and the location of planet formation. Planetary migration of planets near or beyond the snow line may result in a lower than expected moon density which will in turn ensure their removal according to the calculations described herein.

The presence of moons, or lack thereof, in the system is a testable hypothesis given the techniques available from transit variations. A moon will produce TTV and/or Transit Duration Variation (TDV) effects in the timing and shape of the transit photometry, depending on the mass and separation of the moon (Sartoretti \& Schneider 1999; Kipping 2009). Additionally, the size of the moon will play a major role in its detectability in the photometric data. For example, the radius ratio of the Earth's moon and the Earth is 0.272 ; thus the Earth's moon has a transit depth that is $7.4 \%$ as large as the Earth's. Such a deviation would have been detected in the precision Spitzer photometry presented by Gillon et al. (2017), and so moons of comparable size ratios are likely not present, consistent with the findings of this work. Moons as small as the minimum size to be round (radius of $200-300 \mathrm{kms}$ ) are unlikely to be detected without exceptional circunstances, such as phase folding planetary transits for a low-mass star with relatively low stellar activity (Heller 2014). Longterm monitoring with current and future facilities, such as thirty-meter class ground-based telescopes and the James Webb Space Telescope, will place further constraints on the presence of moons in these system.

\section{ACKNOWLEDGEMENTS}

The author would like to thank Duncan Forgan, René Heller, and Natalie Hinkel for their insightful feedback on this work. This research has made use of the NASA Exoplanet Archive, which is operated by the California Institute of Technology, under contract with the National Aeronautics and Space Administration under the 
Exoplanet Exploration Program. The results reported herein benefited from collaborations and/or information exchange within NASA's Nexus for Exoplanet System
Science (NExSS) research coordination network sponsored by NASA's Science Mission Directorate.

\section{REFERENCES}

Agol, E., Steffen, J., Sari, R., Clarkson, W. 2005, MNRAS, 359, 567

Barnes, J.W., O'Brien, D.P. 2002, ApJ, 575, 1087

Barnes, J.W., Fortney, J.J. 2004, ApJ, 616, 1193

Becker, J.C., Adams, F.C. 2017, MNRAS, in press (arXiv:1702.07714)

Domingos, R.C., Winter, O.C., Yokoyama, T. 2006, MNRAS, 373,1227

Forgan, D., Kipping, D. 2013, MNRAS, 432, 2994

Funk, B., Wuchterl, G., Schwarz, R., Pilat-Lohinger, E., Eggl, S. 2010, A\&A, 516, 82

Gillon, M., Jehin, E., Lederer, S.M., et al. 2016, Nature, 533, 221

Gillon, M., Triaud, A.H.M.J., Demory, B.-O., et al. 2017, Nature, 542, 456

Gong, Y.-X., Zhou, J.-L., Xie, J.-W., Wu, X.-M. 2013, ApJ, 769, L14

Hands, T.O., Alexander, R.D., Dehnen, W. 2014, MNRAS, 445, 749

Heller, R. 2012, A\&A, 545, L8

Heller, R., Barnes, R. 2013, AsBio, 13, 18

Heller, R. 2014, ApJ, 787, 14

Heller, R., Williams, D., Kipping, D., et al. 2014, AsBio, 14, 798

Hinkel, N.R., Kane., S.R. 2013, ApJ, 774, 27

Holman, M.J., Wiegert, P.A. 1999, AJ, 117, 621

Holman, M.J., Murray, N.W. 2005, Science, 307, 1288

Kane, S.R., von Braun, K. 2008, ApJ, 689, 492

Kane, S.R., Hinkel, N.R., Raymond, S.N. 2013, AJ, 146, 122

Kipping, D.M. 2009, MNRAS, 392, 181

Kipping, D.M., Schmitt, A.R., Huang, X., et al. 2015, ApJ, 813, 14

Kopparapu, R.K., Ramirez, R., Kasting, J.F., et al. 2013, ApJ, 765,131

Kopparapu, R.K., Ramirez, R.M., SchottelKotte, J., et al. 2014, ApJ, 787, L29
Lasker, J., Joutel, F., Robutel, P. 1993, Nature, 361, 615

Li, G., Batygin, K. 2014, ApJ, 790, 69

Lissauer, J.J., Fabrycky, D.C., Ford, E.B., et al. 2011, Nature, 470,53

Lissauer, J.J., Barnes, J.W., Chambers, J.E. 2012, Icarus, 217, 77

Lissauer, J.J., Jontof-Hutter, D., Rowe, J.F., et al. 2013, ApJ, 770,131

Luger, R., Sestovic, M., Kruse, E., et al. 2017, NatAs, submitted (arXiv:1703.04166)

MacDonald, M.G., Ragozzine, D., Fabrycky, D.C., et al. 2016, AJ, 152, 105

Miralda-Escudé, J. 2002, ApJ, 564, 1019

Namouni, F. 2010, ApJ, 719, L145

Payne, M.J., Deck, K.M., Holman, M.J., Perets, H.B. 2013, ApJ, $775, \mathrm{~L} 44$

Plavchan, P., Werner, M.W., Chen., C.H., et al. 2009, ApJ, 698, 1068

Raymond, S.N., Barnes, R., Veras, D., et al. 2009, ApJ, 696, L98

Reynolds, R.T., McKay, C.P., Kasting, J.F. 1987, AdSpR, 7, 125

Sasaki, T., Barnes, J.W., O'Brien, D.P. 2012, ApJ, 754, 51

Sartoretti, P., Schneider, J. 1999, A\&AS, 134, 553

Sasaki, T., Barnes, J.W. 2014, IJAsB, 13, 324

Spalding, C., Batygin, K., Adams, F.C. 2016, ApJ, 817, 18

Tomasella, L, Marzari, F., Vanzani, V. 1996, P\&SS, 44, 427

Weidner, C., Horne, K. 2010, A\&A, 521, A76

Williams, D.M., Kasting, J.F., Wade, R.A. 1997, Nature, 385, 234

Wolf, E.T. 2017, ApJL, submitted (arXiv:1703.05815)

Xie, J.-W. 2013, ApJS, 208, 22

Zhang, K., Hamilton, D.P., Matsumura, S. 2013, ApJ, 778, 6

Zuluaga, J.I., Kipping, D.M., Sucerquia, M., Alvarado, J.A. 2015, ApJ, 803, L14 